Technological University Dublin

DƯBLIN

ARROW@TU Dublin

2012-05-01

\title{
Real Time Shrinkage Studies in Photopolymer Films Using Holographic Interferometry
}

\author{
Mohesh Moothanchery \\ Technological University Dublin, moheshm@gmail.com \\ Izabela Naydenova \\ Technological University Dublin, izabela.naydenova@tudublin.ie \\ Viswanath Bavigadda \\ Technological University Dublin
}

See next page for additional authors

Follow this and additional works at: https://arrow.tudublin.ie/cieocon2

Part of the Materials Science and Engineering Commons, and the Optics Commons

\section{Recommended Citation \\ Moothanchery, M. et al. (2012) Real-time shrinkage studies in photopolymer films using holographic interferometry. Proc. SPIE 8437, 84370l. Brussels Belgium. 16-19 April. http://dx.doi.org/10.1117/} 12.922413

This Conference Paper is brought to you for free and open access by the Centre for Industrial and Engineering Optics at ARROW@TU Dublin. It has been accepted for inclusion in Conference Papers by an authorized administrator of ARROW@TU Dublin. For more information, please contact arrow.admin@tudublin.ie, aisling.coyne@tudublin.ie,gerard.connolly@tudublin.ie. Funder: DIT Fiosraigh Scholarship Programme

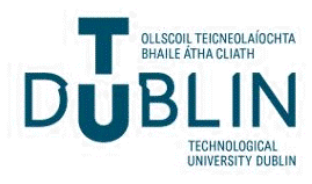




\section{Authors}

Mohesh Moothanchery, Izabela Naydenova, Viswanath Bavigadda, Suzanne Martin, and Vincent Toal 


\title{
Real time shrinkage studies in photopolymer films using holographic interferometry
}

\author{
M. Moothanchery*a, I. Naydenova ${ }^{\mathrm{a}, \mathrm{b}}$, V. Bavigadda ${ }^{\mathrm{a}}$, S. Martin ${ }^{\mathrm{a}}$, V.Toal $^{\mathrm{a}}$ \\ ${ }^{a}$ Centre for Industrial \& Engineering Optics, Dublin Institute of Technology, Dublin 8 , Ireland; \\ ${ }^{\mathrm{b}}$ School of Physics, Dublin Institute of Technology, Dublin 8, Ireland \\ *Corresponding author: mohesh.moothanchery@mydit.ie
}

\begin{abstract}
Polymerisation induced shrinkage is one of the main reasons why photopolymer materials are not more widely used for holographic applications. The aim of this study is to evaluate the shrinkage in an acrylamide photopolymer layer during holographic recording using holographic interferometry. Shrinkage in photopolymer layers can be measured by real time capture of holographic interferograms during holographic recording. Interferograms were captured using a CMOS camera at regular intervals. The optical path length change and hence the shrinkage were determined from the captured fringe patterns. It was observed that the photopolymer layer shrinkage is in the order of $3.5 \%$.
\end{abstract}

Keywords: Holography, interferometry, photopolymer, shrinkage

\section{INTRODUCTION}

Photopolymers are under investigation for applications including LCD displays [1], holographic data storage [2-4], and holographic optical elements [5], because of their easy processing, high photosensitivity, relatively high refractive index contrast and reasonable cost. Polymerisation induced shrinkage is one of the main reasons why photopolymer materials are not more widely used for holographic applications. The aim of this study is to evaluate directly the shrinkage during holographic recording in an acrylamide photopolymer developed at the Centre for Industrial and Engineering Optics [6] using real time holographic interferometry, a non-destructive technique that measures small static or dynamic changes occurring in an object [7-9]. Shrinkage in photopolymer layers has been determined [10] by measuring the shift in the angular position of the Bragg peak. The disadvantage of the Bragg curve measurement is that the shrinkage can be measured only after recording the grating whereas real-time holographic interferometry is a dynamic method by which deformations, including photo induced dimensional changes, in a material can be monitored throughout the entire experiment [11].

Holographic interferometry was introduced in 1965 by Powell and Stetson [12]. Using the interferometric technique shrinkage in photopolymer layers can be determined by real-time capture of holographic interferograms during holographic recording. The interferograms are produced by interference between a holographically reconstructed light wave traversing the photopolymer layer or reflected from its surface, recorded in a separate hologram before photopolymerization begins and a wave traversing or reflected from the photopolymer surface during the photopolymerization process, which is insensitive to the hologram recording light. A Virtual instrument (VI) developed in LabVIEW (Version 8.2) was used to capture interferograms using a CMOS camera at regular intervals. One can determine the optical path change and hence the shrinkage from the captured fringe patterns. The important feature of holographic interferometry is that the fringes corresponding to the displacement of the object are produced in real-time. The fringes produced in such a way will provide useful information regarding changes in the dimensions of the photopolymer.

Real time holographic interferometry helps to determine minute changes in an object in real time by superimposing the image reconstructed from the hologram of the object, on the object itself while it is undergoing change.

The vector displacement $(L)$ at a point on the surface of the object can be determined using holographic interferometry, since the vector displacement is proportional to the phase difference between two interfering beams [13]. The phase difference $\phi$ is given by 


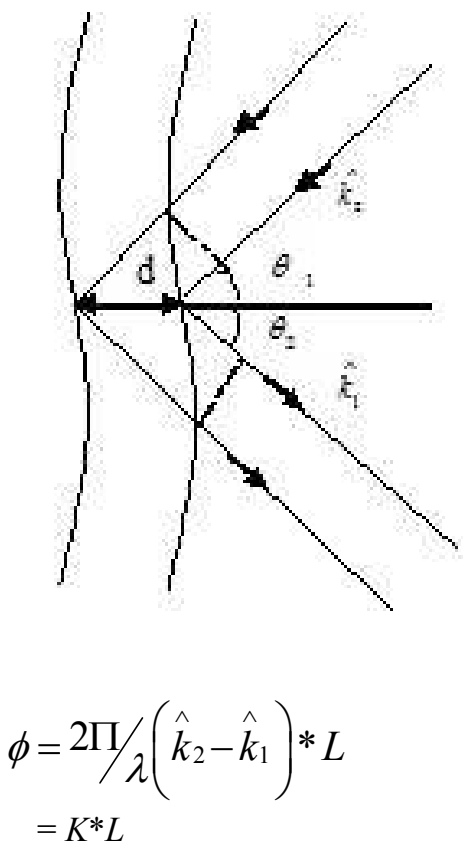

Where $\hat{k}_{1}$ and $\hat{k}_{2}$ are unit vectors in the direction of illumination and observation. $K$ is known as the sensitivity vector $[14,15]$.

The optical path length with respect to angles of illumination and observation is

$$
O P D=d\left(\cos _{\theta 1}+\cos _{\theta 2}\right)
$$

where $\theta_{1}$ and $\theta_{2}$ are the angle of illumination and observation respectively. The optical path length can also be expressed in terms of wavelength $\lambda$

$$
O P D=n \lambda
$$

Where $n$ is a number. From equation (2) and (3) the object displacement $d$ is

$$
d=n \lambda /\left(\cos _{\theta 1}+\cos _{\theta 2}\right)
$$

\section{EXPERIMNTAL PROCEDURES}

\subsection{Sample preparation}

A green sensitive photopolymer layer was prepared as described in [6]. Briefly, $0.6 \mathrm{~g}$ of acrylamide monomer was added to $17.5 \mathrm{ml}$ stock solution of polyvinyl alcohol $(20 \% \mathrm{wt})$. Then $2 \mathrm{ml}$ of triethanolamine was added. To this solution $0.2 \mathrm{~g}$ of $N, N$-methylene bisacrylamide and finally $4 \mathrm{ml}$ of Erythrosine-B dye was added $(0.11 \%$ wt. water stock solution $)$. Of this photopolymer solution, volumes of $0.25,0.5$ and $0.75 \mathrm{ml}$ were spread on $25 \mathrm{~mm} \times 75 \mathrm{~mm}$ glass plates. The samples were allowed to dry for 24 hours. The corresponding sample thicknesses after drying were approximately 50,100 and $150 \mu \mathrm{m}$ respectively. A solution was also made up using similar amounts of acrylamide, methylenebisacrylamide, triethnolamine, polyvinyl alcohol $(10 \% \mathrm{wt})$ and $4 \mathrm{ml}$ of methylene blue dye. Of this solution $0.6 \mathrm{ml}$ was spread on a 25 $\mathrm{mm} \times 75 \mathrm{~mm}$ glass plate and allowed to dry for 24 hours. The corresponding layer thickness after drying was approximately $60 \mu \mathrm{m}$. Theses red sensitized layers were used to record holograms of the studied green sensitive layers. 


\subsection{Experimental technique}

The interferometer setup is as shown in Fig.1. It comprises two independent pairs of interfering beams. The green $(532 \mathrm{~nm})$ beams interfere at a green sensitive photopolymer (Er.B sample) to produce a grating. The red $(633 \mathrm{~nm})$ beams are used in an interferometry arrangement to monitor movement in the surface of that grating. The $633 \mathrm{~nm}$ beam from a $\mathrm{He}-\mathrm{Ne}$ Laser is spatially filtered collimated and split into two beams using a beam splitter. One of the beams is partially reflected from the green sensitive, Erythrosine B sample (object beam) whereas the other beam (reference beam) is partially reflected from a glass plate and these beams are allowed to interfere so as to record a hologram in the red sensitive Methylene Blue layer of the surface of the of Erythrosine B (Er. B) layer. The angles of illumination and observation of the object beam were $26.2^{\circ}$ and $33.7^{\circ}$ respectively. The combined intensity of the $633 \mathrm{~nm}$ beam, the light reflected from the Er.B surface and from the glass plate was $0.5 \mathrm{~mW} \mathrm{~cm}$. After recording the hologram in the red sensitive layer for around $140 \mathrm{sec}$ the beam from a Torus $532 \mathrm{~nm}$ laser, spatially filtered, and split into two beams using a beam splitter was switched on. These beams interfere with one another to record a holographic grating in the Erythrosine. B layer as a result of which the material shrinks. The combined intensity from the $532 \mathrm{~nm}$ beams used to record the grating was $5 \mathrm{~mW} \mathrm{~cm}^{-2}$. Due to shrinkage, a change occurs in the optical path of the red beam reflected from the surface of the green sensitive layer and this can be calculated from the number of fringes appearing as a result of the interference of the beam reflected from the green sensitive layer surface and the beam reconstructed by the hologram recorded in the red sensitive layer. The appearance of fringes can be observed during the recording of the holographic grating in the green sensitive layer.

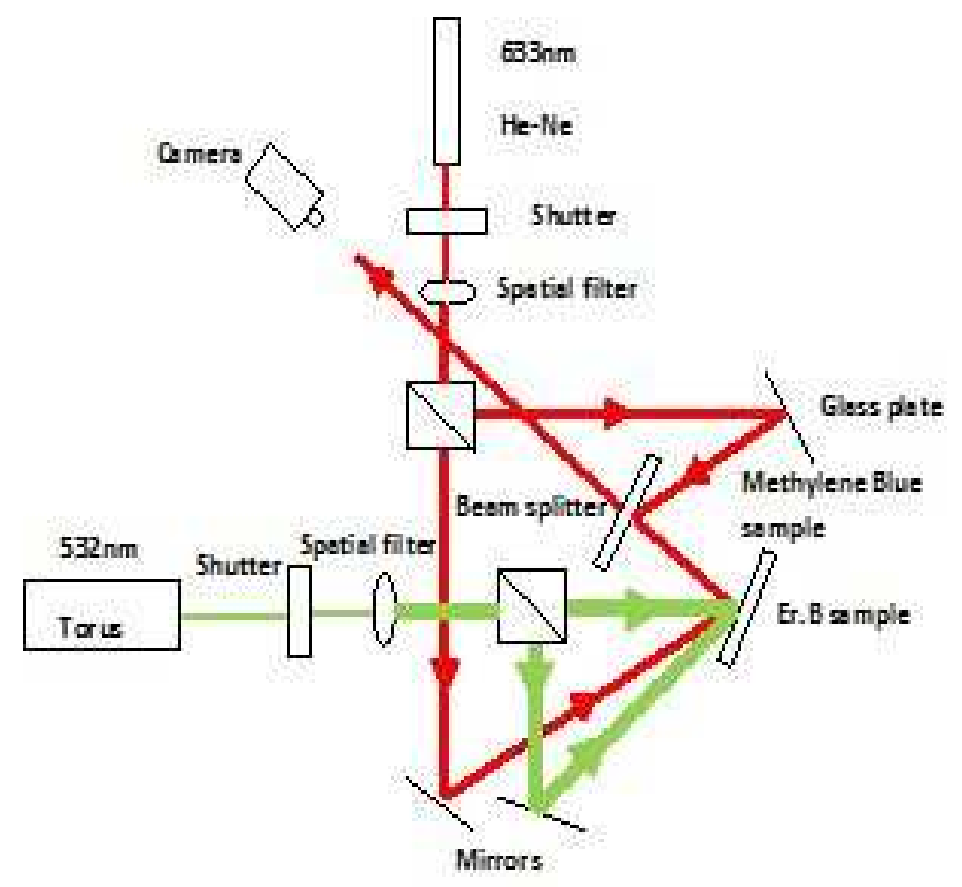

Figure.1 Holographic Interferometric System

Due to limited visibility of holographic interferometry fringes, in order to determine photopolymer shrinkage we have used image subtraction. A CMOS camera was used. A LabVIEW (Version 8.2) coded virtual instrument (VI) was used with a National Instruments IMAQ-1409 frame grabber card to capture images. The camera (AVT Guppy F-036B) was set in externally triggered mode and supplied by a D/A board with a digital pulse to initiate each image capture. The digital pulse frequency can be varied according to our requirement (1 to $25 \mathrm{~Hz}$ ). A reference frame was subtracted from each incoming frame and the result representing the change in the interferogram displayed on the computer monitor in real time. The reference frame and other acquired frames were also stored in the computer. The movement of the interference fringes was captured as a live video which was processed using NI Vision Assistant software. The 
advantage of the subtraction method for the production of secondary fringes is that it can produce high contrast [16]. The reference frame was captured at the end of the recording of the hologram in the red sensitive layer, just before switching on the green laser.

\section{EXPERIMENTAL RESULTS}

A hologram of the front surface of the $90 \mu \mathrm{m}$ thick Er. B layer was recorded in a $60 \mu \mathrm{m}$ Methylene Blue sensitive layer using the interference of a $633 \mathrm{~nm}$ beam reflected from the front surface of the Er.B layer and a reference beam reflected from a glass plate. The image reconstructed from this hologram, Fig. 2(a), was used as the reference image for the static subtraction method. It was captured after the recording process at $633 \mathrm{~nm}$ was completed and the Methylene Blue sensitive photopolymer layer was fully polymerised. When the Er.B sample was not undergoing polymerisation no fringes were produced by the subtraction of the reference image, Fig.2 (b). The result of the subtraction was observed for several seconds, confirming that the fringes, appearing at this stage are produced only while recording a holographic grating. The interferogram obtained while the Er. B layer was undergoing polymerization using the 532 beam is shown in Fig.2(c). Fig. 2(c) clearly shows a dark fringe and the number of fringes appearing will vary depending on the layer thickness and the duration of polymerisation.

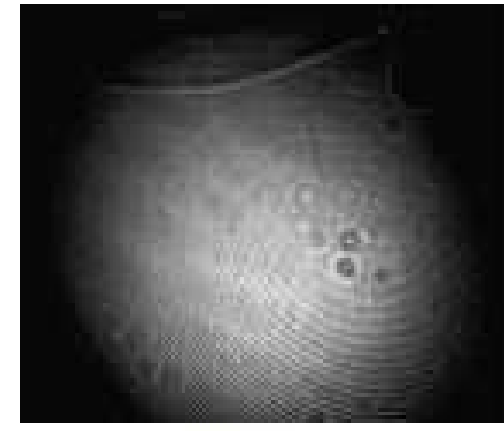

(a)

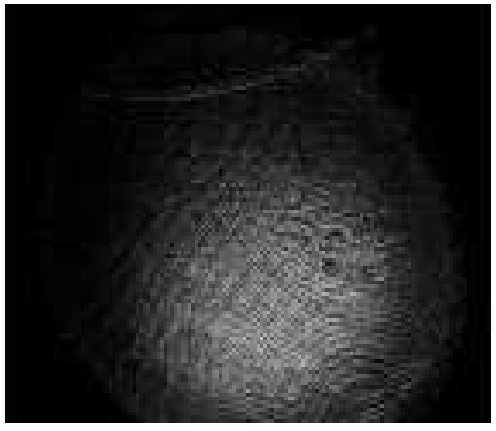

(b)

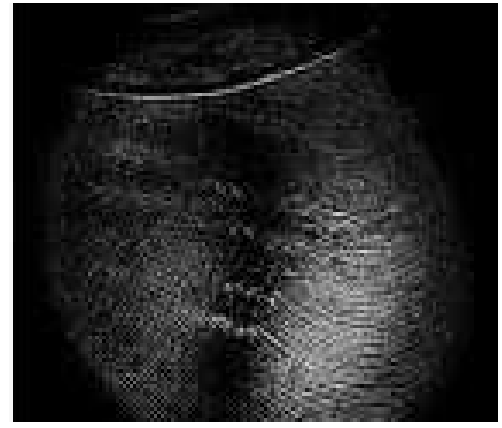

(c)

Figure.2 a) Reference frame, produced by capturing the beam reconstructed by the hologram recorded in the red sensitive layer; b) Result of subtraction of the reference frame from the current frame ( before switching on the green laser); c) Result of subtraction of the reference frame from the current frame ( after switching on the green laser);

Table 1. Shows the total number of fringes appearing during the recording and hence the shrinkage for layer thicknesses of 55,90 and $155 \mu \mathrm{m}$ for exposure time of $180 \mathrm{sec}$.

Table.1: Thickness dependence on shrinkage

\begin{tabular}{|l|l|l|l|}
\hline Thickness $(\boldsymbol{\mu} \mathbf{m})$ & No: of fringes (n) & Path difference (d) $\boldsymbol{\mu} \mathbf{m}$ & Shrinkage (\%) \\
\hline 55 & 6 & 2.21 & 4 \\
\hline 90 & 9 & 3.31 & 3.68 \\
\hline 155 & 15 & 5.52 & 3.56 \\
\hline
\end{tabular}

From the table it is clear that the absolute shrinkage increases with photopolymer layer thickness. 


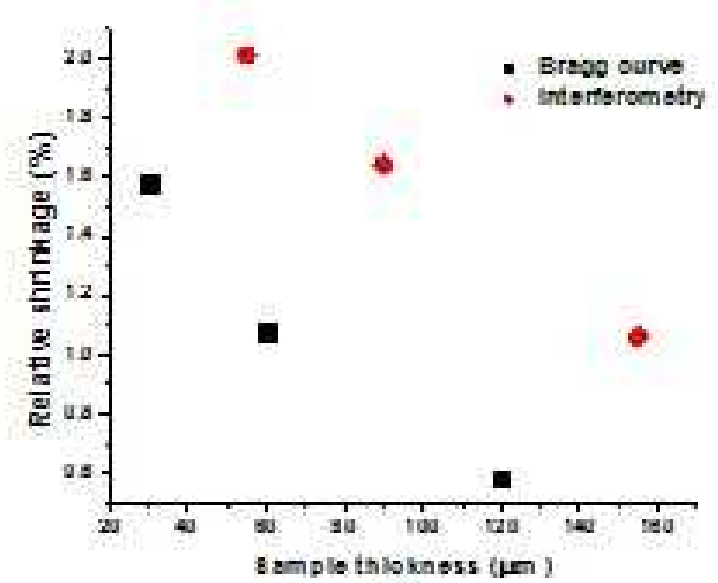

(a)

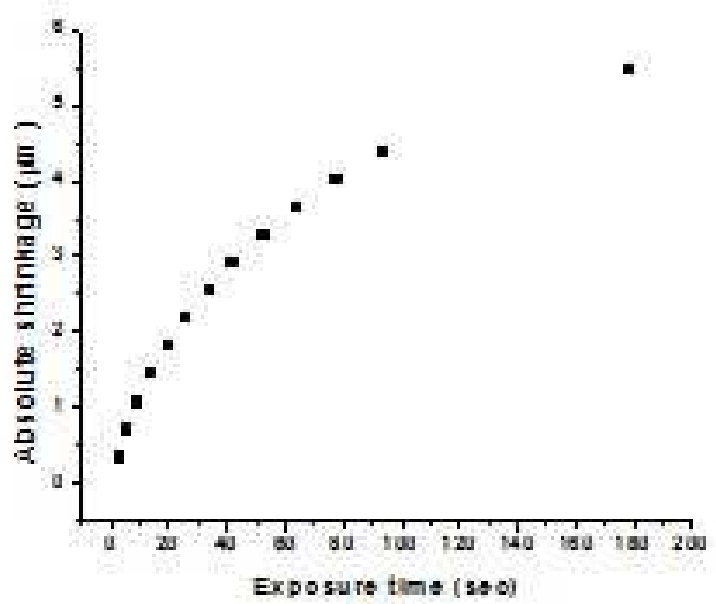

(b)

Figure.3 a) Comparative study of relative shrinkage with sample thickness; b) Real time shrinkage of $155 \mu \mathrm{m}$ layer with exposure time

Figure.3(a) shows a comparison of relative shrinkage versus sample thickness using Bragg technique[10] and holographic interferometry technique. Shrinkage measurements using Bragg curve shift were made for sample thicknesses of 30, 60 and $120 \mu \mathrm{m}$ and the gratings were recorded at $10 \mathrm{~mW} \mathrm{~cm}^{-2}$ and exposure time of $8 \mathrm{sec}$ whereas in the holographic interferometric technique shrinkage has been calculated at the end of $16 \mathrm{sec}$ from the real time curve of shrinkage with exposure, such as that shown in fig. 3(b). The recording intensity was $5 \mathrm{~mW} \mathrm{~cm}^{-2}$. Both sets of results show the same trend. The shrinkage at $16 \mathrm{sec}$ has been taken for the comparison to keep the exposure energy constant at $80 \mathrm{~mJ} \mathrm{~cm}^{-2}$. Greater shrinkage has been observed for recording with lower intensity and longer exposure time [10]. Fig. 3(b) shows the absolute shrinkage measurements in real time using the holographic interferometric technique for sample thickness $155 \mu \mathrm{m}$. The results show that shrinkage increases correspondingly with exposure time. We have observed that the polymerisation shrinkage occurs faster during the early stage of exposure.

\section{CONCLUSIONS}

Real time holographic interferometry has been used to characterise the process of shrinkage in acrylamide based photopolymer during holographic recording. Initial tests were carried out in order to confirm that the observed interference fringes are the result of the shrinkage during the holographic recording. The absolute shrinkage in the layer is proportional to the layer thickness. Comparison is made with data previously obtained using the Bragg shift method.

\section{ACKNOWLEDGEMENT}

This work is supported by DIT Fiosraigh Research Scholarship programme. The authors would like to thank the School of Physics DIT and the Focas Research Institute for technical support.

\section{REFERENCES}

[1] Biles,J., "Holographic Color Filters for LCDs", SID 94 Digest, 403-406 (1994).

[2] $\mathrm{Pu}, \mathrm{A}$. and Psaltis,D, "High-density recording in photopolymer based holographic three-dimensional disks," Appl. Opt. 35, 2389-2398 (1996). 
[3] Rhee, U.S., Caulfield, H.J., Shamir,J., Vikram,C.S.and Mirsalehi,M.M., "Characteristics of the DuPont photopolymer for angularly multiplexed page-oriented holographic memories," Opt. Eng. 32, 1839-1847(1993).

[4] Naydenova,I, Sherif,H, Mintova,S., Martin,S. and Toal,V. "Holographic recording in nanoparticle doped photopolymer," Proc. SPIE 6252, 625206 (2006).

[5] Hemmer,P., Shahriar,S., Ludman,J and Caulfield,H.J., "Holographic Optical Memories," in "Holography for the New Millennium" eds. J. Ludman, H. J. Caulfield, and J. Riccobono, Springer-Verlang Newyork, 179-189 (2002).

[6] Martin,S., Feely,C.A. and Toal,V., "Holographic recording characteristics of an acrylamide based photopolymer," Appl. Opt. 36, 5757-5768, (1997).

[7] Manley, M.T., Ovryn, B., Stern, L.S., "Evaluation of double-exposure holographic interferometry for biomechanical measurements in vitro," J Orthop Res, 5 144-9. (1997

[8] Vest, C.M, "Holographic Interferometry" Wiley, New York (1979).

[9] Rastogi, K. ed., Holographic interferometry: Principles and Methods, Springer Series in Optical Sciences, 1 Springer-Verlag Berlin, 68, (1994)

[10] Moothanchery, M., Naydenova, I., and Toal,V., "Study of the shrinkage caused by holographic grating formation in acrylamide based photopolymer film," Optics Express. 19, 13395-13404 (2011)

[11] Pantelić,D., Blažić,L., Savić-Šević,S., Murić,B., Vasiljević,D., Panić,B., Belić,I., "Real-time measurment of internal stress of dental tissue using holography,”. Optics Express, 15 6823-6830, (2007).

[12] Powell \& Stetson, "Interferometric vibration analysis by wavefront reconstruction." JOSA, 55, 1593-1598 (1965)

[13] Hariharan, P., Oreb, B.F. and Brown, N., "Real-time holographic interferometry: a microcomputer system for the measurement of vector displacements," APPLIED OPTICS, 22, 876-880 (1983)

[14] Aleksandrov. E. B. and Bonch-Bruevich. A. M Sov. Phys. Tech.Phys. 12, 258 (1967).

[15] Ennos. A. E, "Measurement of in plane surface strain by hologram interferometry," J. Phys. E, 1, 731-734 (1968)

[16] Bavigadda.V, Jallapuram.R, Mihaylova.E, and Toal.V, "Electronic speckle-pattern interferometer using holographic optical elements for vibration measurements", Optics Letters, 35, 3273-3275 (2010) 